\title{
Myeloid malignancies with $5 q$ and $7 q$ deletions are associated with extreme genomic complexity, biallelic TP53 variants, and very poor prognosis
}

\author{
Beth A. Pitel $\mathbb{C}^{1}$, Neeraj Sharma', Cinthya Zepeda-Mendoza ${ }^{1,8}$, James B. Smadbeck², Kathryn E. Pearce', \\ Joselle M. Cook ${ }^{3}$, George Vasmatzis ${ }^{2}$, Zohar Sachs $\mathbb{1}^{4}$, Rashmi Kanagal-Shamanna $\mathbb{0}^{5}$, David Viswanatha ${ }^{6}$, Sheng Xiao ${ }^{7}$, \\ Robert B. Jenkins ${ }^{1}$, Xinjie Xu', Nicole L. Hoppman', Rhett P. Ketterling ${ }^{1}$, Jess F. Peterson', Patricia T. Greipp ${ }^{1}$ and \\ Linda B. Baughn (10'
}

Dear Editor,

Acute myeloid leukemia (AML) is an aggressive myeloid neoplasm representing the most common type of acute leukemia in adults ${ }^{1,2}$. AML is classified into multiple genetic subtypes based on recurrent structural variations (SVs), copy number variations (CNVs), aneuploidies, and single nucleotide variants (SNVs). These genetic subtypes inform prognosis and influence clinical management ${ }^{3-6}$.

Cytogenetically visible deletions of chromosomes 5 and/ or 7 in the absence of a WHO-defined recurrent SV represent a distinct subgroup associated with complex karyotype, pathogenic TP53 variants, and adverse prognosis ${ }^{4}$. Identification of this subtype relies upon conventional cytogenetic techniques such as chromosome studies and/or fluorescence in situ hybridization (FISH) ${ }^{4}$. However, structural genomic complexity may be grossly underestimated and imprecise by these conventional chromosome studies and limited information is retrieved from FISH testing due to the targeted nature of the assay ${ }^{7}$. While genomic complexity has been explored using chromosomal microarray studies ${ }^{8,9}$, structural complexity profiling in AML by next-generation sequencing (NGS) has been largely unexplored. The objective of this study was to explore the relationship between $5 \mathrm{q}$ and $7 \mathrm{q}$ deletions, genome-wide genomic complexity as determined by

\footnotetext{
Correspondence: Linda B. Baughn (baughn.linda@mayo.edu)

${ }^{1}$ Department of Laboratory Medicine and Pathology, Division of Laboratory

Genetics and Genomics, Mayo Clinic, Rochester, MN, USA

${ }^{2}$ Center for Individualized Medicine-Biomarker Discovery, Mayo Clinic,

Rochester, MN, USA

Full list of author information is available at the end of the article

These authors contributed equally: Beth A. Pitel, Neeraj Sharma
}

NGS and conventional chromosome studies, TP53 variants, and disease outcome.

\section{Study characteristics}

Following Mayo Clinic Institutional Review Board approval, we searched our Mayo Clinic Genomics database from 2017 and 2018 to identify patients that had a myeloid clone with a $5 \mathrm{q}$ deletion and/or $7 \mathrm{q}$ deletion identified by FISH and/or chromosomes studies (including cases with monosomies of chromosomes 5 and/or 7). We also identified cases with a normal karyotype (NK) AML clone as a control for low genomic complexity identified by conventional cytogenetics. A total of 103 cases had either a NK (NK, $N=52)$, 7q deletion (7q del, $N=12$ ), $5 \mathrm{q}$ deletion ( $5 \mathrm{q}$ del, $N=19$ ), or $5 \mathrm{q}$ deletion and $7 \mathrm{q}$ deletion $(5 \mathrm{q} / 7 \mathrm{q}$ del, $N=20)$ [Fig. $1 \mathrm{~A}$, Table S1].

The majority of cases, 90 (87\%) represented diagnostic specimens and 13 (13\%) were relapsed AML. Specifically, 48 (47\%) were de novo AML, 37 (36\%) were secondary AML (31 had AML with myeloid-related changes (MRC) and 6 were therapy-related). We also included five additional high-grade myeloid neoplasms with $5 \mathrm{q}$ del and/or $7 \mathrm{q}$ del including three patients with MDS (one with therapy-related MDS with $6 \%$ blasts, two with MDS with excess blasts; $12-20 \%$ blasts and $10-15 \%$ blasts) and two cases with an unspecified myeloid malignancy [Table S1, Table S2]. The median age was 68 years (range 9-90) with a slight male predominance of 53\% [Table S1]. The European Leukemia Net (ELN) 2017 prognostication of patients in the NK subgroup depended largely on their $\mathrm{SNVs}^{4}$ [Table S1]. Nineteen (37\%) NK samples could not be stratified due to incomplete sequencing data. Of $33 \mathrm{NK}$ 


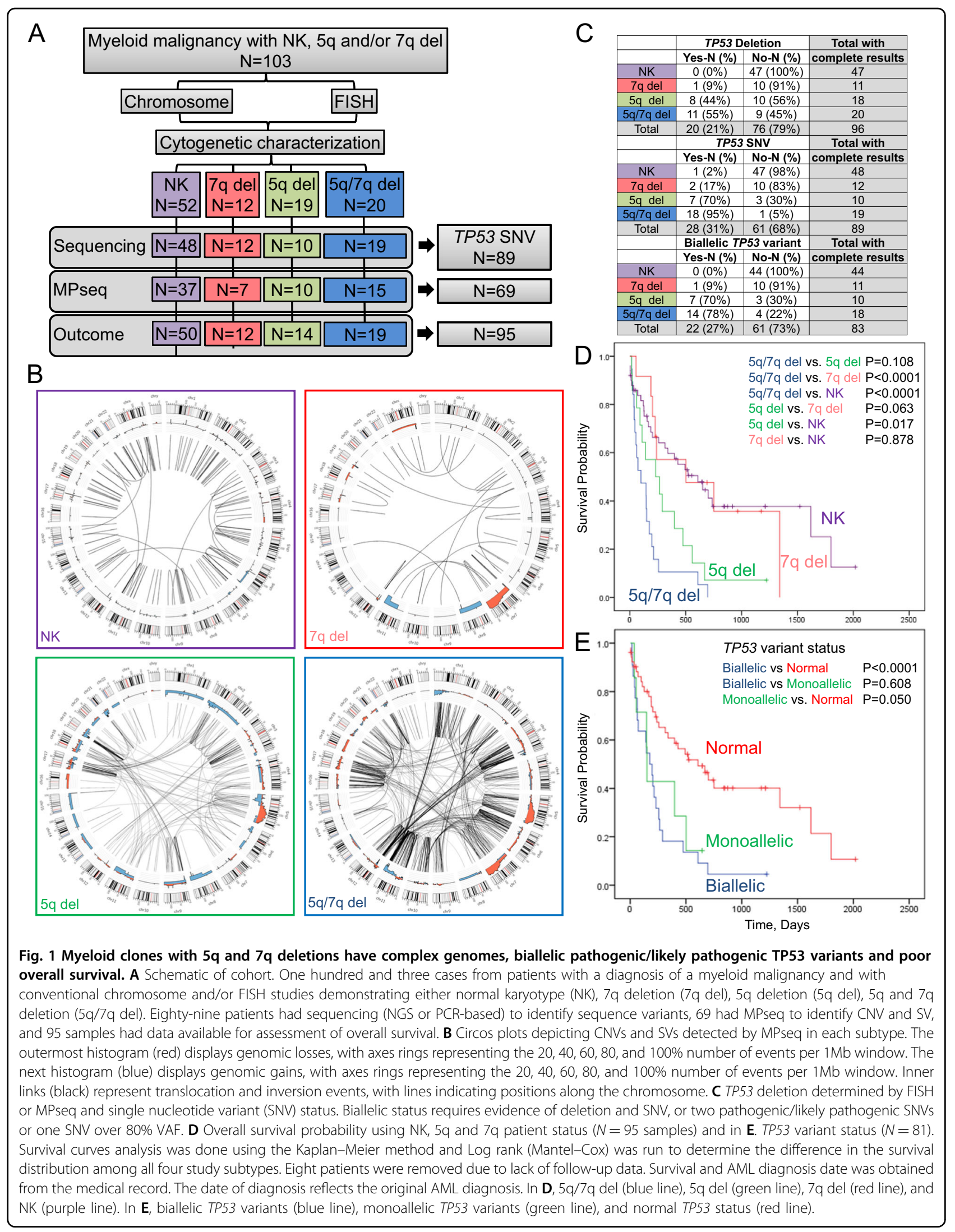


cases with ELN prognostication data, 10 were favorable, 11 were intermediate, and 12 were adverse. Of the remaining 51 cases that did not have NK, 48 cases had adverse risk due to identification of monosomy $5,5 q \mathrm{del}$, monosomy 7, complex karyotype and/or pathogenic/ likely pathogenic variants (deletions or SNV) [Table S1]. While monosomy 7 is classified as high risk by ELN, 7q dels are classified as intermediate risk in the absence of other high-risk abnormalities. Two cases were classified as intermediate with a 7q del, a non-complex karyotype and no high-risk variants. One case had a 7q del, but evaluation for high-risk SNVs was incomplete [Table S1].

Sixty-nine cases had available DNA from bone marrow (BM) or peripheral blood (PB) for analysis by mate-pair sequencing (MPseq), a form of NGS optimized for the detection of SVs and $\mathrm{CNVs}^{7}$. Additional materials and methods details are in "Supplementary Information". By MPseq, the minimum deleted region of chromosome $5 \mathrm{q}$ was $\sim 6 \mathrm{Mb}$ from $5 \mathrm{q} 31.1$ to $5 \mathrm{q} 31.2$ (chr5:134132000-139782000 [GRCh38]), encompassing EGR1, and the minimum deleted region of chromosome $7 \mathrm{q}$ was $\sim 10 \mathrm{Mb}$ from $7 \mathrm{q} 32.1$ to $7 \mathrm{q} 34$ (chr7:128933000-138962000 [GRCh38]) [Fig. 1B, Fig. S1]. No large deletions in the critical regions of chromosome $5 \mathrm{q}$ and $7 \mathrm{q}$ were identified by MPseq in NK samples [Fig. 1B, Fig. S1]. Genome-wide SVs and CNVs demonstrated overall increased genomic complexity of $5 q$ del and $5 q / 7 q$ del subtypes in comparison to NK and 7q del subtypes, with the greatest genomic complexity identified in the $5 q / 7 q$ del subtype [Fig. 1B]. The median number of genome-wide $\mathrm{CN}$ gains, CN losses, and SVs were lower in NK (2.0, 5.0, and $4.0)$ and $7 \mathrm{q}$ del (2.0, 8.0, and 6.0) and higher in $5 \mathrm{q}$ del (11.5, 14.5, and 17.5) and 5q/7q del (14.0, 24.0, and 60.0), a difference that was significant among the 4 subtypes in each category $(p<0.001)$ [Table S3]. There was also an increased overall copy number burden (CNB) in cases with $5 \mathrm{q} / 7 \mathrm{q}$ del, even when excluding any $\mathrm{CN}$ abnormalities involving $5 \mathrm{q}$ and 7q [Table S4]. Overall CNB correlated with karyotype complexity determined from the conventional chromosome results [Fig. S2]. Most 5q del and 5q/7q del subtypes were characterized by chromoplexy, chromothripsis, or progressive complexity with enrichment of SV involving chromosomes 5, 12, and 17, features absent in NK and 7q del cases [Fig. S3].

We next evaluated the incidence of pathogenic/likely pathogenic TP53 variants (deletions and SNVs). TP53 deletions were identified in 20/96 (21\%) cases [Fig. 1C, Fig. S4]. None of the NK subtypes had a TP53 deletion, 1 (9\%) 7q del, 8 (44\%) 5q del, and 11 (55\%) 5q/7q del cases had a TP53 deletion. Pathogenic/likely pathogenic TP53 SNVs were identified in 28/89 (31\%) cases. One NK case had a TP53 SNV ( 5\% VAF), 2 (17\%) 7q del, 7 (70\%) 5q del, and 18 (95\%) 5q/7q del had TP53 SNVs. Monoallelic TP53 variants were found in 7/83 (8\%) cases and biallelic TP53 variants were found in 22/83 (27\%) of cases [Fig. 1C,
Fig. S4]. Biallelic TP53 variants were predominantly identified in cases with $5 \mathrm{q}$ del $(70 \%)$ and $5 \mathrm{q} / 7 \mathrm{q}$ del $(78 \%)$. Fourteen of 16 cases (88\%) with a TP53 monoallelic deletion that were evaluable for TP53 SNV had a TP53 SNV. Two samples with a TP53 deletion did not have a TP53 SNV demonstrating that TP53 deletion status is often predictive of a TP53 SNV on the remaining allele. In contrast, 14 of $28(50 \%)$ cases with a TP53 SNV had a TP53 deletion; the remaining 14 had a TP53 SNV without a TP53 deletion. No TP53 pathogenic/likely pathogenic variants were identified in 5 complex karyotype-AML samples without $5 \mathrm{q}$ del and/or 7q del (data not shown). The type and location of each TP53 SNV are shown in Fig. S5. Since TP53 variants have been reported to associate with chromosome instability in myeloid cells ${ }^{8,10,11}$, cases with TP53 SNVs had a higher median number of $\mathrm{CN}$ gains (14.0 vs. 2.0), CN losses (19.5 vs. 5.0), and SVs (51.0 vs. 4.0) compared to cases with normal TP53, with the greatest fold change (13-fold) was observed in the number of SVs in association with TP53 variants [Fig. S5].

We next evaluated the contribution of $5 \mathrm{q}$ and $7 \mathrm{q}$ deletions, TP53 variant status and genomic complexity on overall survival (OS). The median OS was significantly shorter for patients with $5 \mathrm{q} / 7 \mathrm{q}$ del (100 days, $95 \% \mathrm{CI}$, 0-217 days, $p<0.0001)$ or $5 \mathrm{q}$ del $(231$ days, $95 \% \mathrm{CI}$, $2-460$ days, $p=0.017)$ compared to NK $(608$ days, $95 \% \mathrm{CI}$, 300-918 days) and between $5 \mathrm{q} / 7 \mathrm{q}$ del compared to $7 \mathrm{q}$ del (502 days, $95 \% \mathrm{CI}, 0-1203$ days) $(p<0.0001)$ [Fig. 1D] similar with prior reports ${ }^{11,12}$. No significant difference in OS was observed between $5 q / 7 q$ del and $5 q$ del and between NK and $7 \mathrm{q}$ del. The median OS was also significantly shorter for patients with biallelic (175 days, 95\%CI, 102-247 days, $p<$ $0.0001)$ or monoallelic TP53 variants (150 days, $95 \% \mathrm{CI}$, $140-160$ days, $p=0.050)$ compared to patients with normal TP53 (608 days, 95\%CI, 304-912 days). No significant difference in OS was observed between biallelic and monoallelic TP53 categories, as previously reported $^{8}(p=0.608)$ [Fig. 1E]. Patients with high genomic complexity identified by MPseq and complex and monosomal karyotypes had a significantly shorter median OS compared to patients without these features $(p<0.0001)$ [Fig. S6]. The greatest risk of death was found in $5 \mathrm{q} / 7 \mathrm{q}$ del (univariate risk ratio 3.39, $p<0.0001$; $95 \% \mathrm{CI}: 1.94-5.92$ and multivariate risk ratio 2.58, $p=0.003$; 95\%CI: $1.36-4.88)$ in comparison to cases with only $5 \mathrm{q}$ del (univariate risk ratio $1.61, p=0.124 ; 95 \% \mathrm{CI}$ : $0.88-2.97$ ). Cases with $7 \mathrm{q}$ del (univariate risk ratio $0.68, p=$ 0.306 ; $95 \% \mathrm{CI}$ : $0.32-1.42$ ) and NK (univariate risk ratio 0.46 , $p=0.002$; 95\%CI: $0.28-0.75)$ had reduced risk of death compared to $5 \mathrm{q} / 7 \mathrm{q}$ del [Table S5]. Improved OS of $7 \mathrm{q}$ del cases may be explained by 10 of 12 (83\%) of 7q del cases had a simple karyotype, with $<3$ cytogenetic abnormalities, in contrast to $5 \mathrm{q}$ del or $5 \mathrm{q} / 7 \mathrm{q}$ del cases, similar to previously published observations ${ }^{11}$. Similar OS between the $7 q$ and NK cases may be due to the incorporation of NK cases with 
less favorable ELN risk categories based on mutation status (Table S1), further narrowing the OS gap between the NK and $7 \mathrm{q}$ del cohorts.

In summary, we describe the use of genome-wide NGS in the characterization of genomic complexity in AML, with the potential to reframe our understanding of complex genomic events. To our knowledge, very few studies have specifically evaluated the structural complexity incorporating both CNVs and SVs of AML genomes by NGS ${ }^{13-15}$. Here we show that myeloid malignancies with deletions of $5 q$ and $7 q$ are associated with additional complex genomic findings not appreciated by conventional chromosome studies including increased copy number burden, chromothripsis, chromoplexy, progressive genomic complexity, and very poor overall survival.

\section{Acknowledgements}

This study was supported by funds from the Mayo Clinic Department of Laboratory Medicine and Pathology.

\begin{abstract}
Author details
${ }^{1}$ Department of Laboratory Medicine and Pathology, Division of Laboratory Genetics and Genomics, Mayo Clinic, Rochester, MN, USA. ${ }^{2}$ Center for Individualized Medicine-Biomarker Discovery, Mayo Clinic, Rochester, MN, USA. ${ }^{3}$ Department of Medicine, Division of Hematology, Mayo Clinic, Rochester, MN, USA. ${ }^{4}$ Division of Hematology, Oncology, and Transplantation, Department of Medicine and Masonic Cancer Center, University of Minnesota, Minneapolis, MN, USA. ${ }^{5}$ Department of Hematopathology, University of Texas MD Anderson Cancer Center, Houston, TX, USA. ${ }^{6}$ Department of Laboratory Medicine and Pathology, Division of Hematopathology, Mayo Clinic, Rochester, MN, USA. ${ }^{7}$ Department of Pathology, Brigham and Women's Hospital, Harvard Medical School, Boston, MA, USA. ${ }^{8}$ Present address: Cytogenetics and Genomic Microarray Laboratory, ARUP Laboratories, Salt Lake City, UT, USA
\end{abstract}

\section{Conflict of interest}

Algorithms described in this manuscript are licensed to WholeGenome LLC owned by George Vasmatzis.

\section{Publisher's note}

Springer Nature remains neutral with regard to jurisdictional claims in published maps and institutional affiliations.

Supplementary information The online version contains supplementary material available at https://doi.org/10.1038/s41408-021-00416-4.
Received: 16 October 2020 Revised: 30 December 2020 Accepted: 19 January 2021

Published online: 08 February 2021

\section{References}

1. De Kouchkovsky, I. \& Abdul-Hay, M. 'Acute myeloid leukemia: a comprehensive review and 2016 update'. Blood Cancer J. 6, e441 (2016).

2. Siegel, R. L., Miller, K. D. \& Jemal, A. Cancer statistics, 2020. CA Cancer J. Clin. 70, 7-30 (2020).

3. Arber, D. A. et al. The 2016 revision to the World Health Organization classification of myeloid neoplasms and acute leukemia. Blood. 127, 2391-2405 (2016).

4. Dohner, H. et al. Diagnosis and management of AML in adults: 2017 ELN recommendations from an international expert panel. Blood. 129, 424-447 (2017).

5. Bullinger, L., Dohner, K. \& Dohner, H. Genomics of acute myeloid leukemia diagnosis and pathways. J. Clin. Oncol. 35, 934-946 (2017).

6. Papaemmanuil, E. et al. Genomic classification and prognosis in acute myeloid leukemia. N. Engl. J. Med. 374, 2209-2221 (2016).

7. Aypar, U. et al. Mate pair sequencing improves detection of genomic abnormalities in acute myeloid leukemia. Eur. J. Haematol. 102, 87-96 (2019).

8. Rucker, F. G. et al. TP53 alterations in acute myeloid leukemia with complex karyotype correlate with specific copy number alterations, monosomal karyotype, and dismal outcome. Blood 119, 2114-2121 (2012).

9. Fontana, M. C. et al. Chromothripsis in acute myeloid leukemia: biological features and impact on survival. Leukemia 32, 1609-1620 (2018).

10. Christiansen, D. H., Andersen, M. K. \& Pedersen-Bjergaard, J. Mutations with loss of heterozygosity of p53 are common in therapy-related myelodysplasia and acute myeloid leukemia after exposure to alkylating agents and significantly associated with deletion or loss of 5q, a complex karyotype, and a poor prognosis. J. Clin. Oncol. 19, 1405-1413 (2001).

11. Mrozek, K. et al. Complex karyotype in de novo acute myeloid leukemia: typical and atypical subtypes differ molecularly and clinically. Leukemia $\mathbf{3 3}$, 1620-1634 (2019).

12. Grimwade, D. et al. Refinement of cytogenetic classification in acute myeloid leukemia: determination of prognostic significance of rare recurring chromosomal abnormalities among 5876 younger adult patients treated in the United Kingdom Medical Research Council trials. Blood 116, 354-365 (2010).

13. Mack, E. K. M. et al. Comprehensive genetic diagnosis of acute myeloid leukemia by next-generation sequencing. Haematologica 104, 277-287 (2019).

14. Kim, J. C. et al. Cryptic genomic lesions in adverse-risk acute myeloid leukemia identified by integrated whole genome and transcriptome sequencing. Leukemia 34, 306-311 (2020).

15. Levy, B. et al. A national multicenter evaluation of the clinical utility of optical genome mapping for assessment of genomic aberrations in acute myeloid leukemia. medRxiv. https://doi.org/10.1101/2020.11.07.20227728 (2020). 